\title{
Delta Excitation in the Compressed Finite Nucleus ${ }^{90} \mathrm{Zr}$
}

\author{
Mohammed Hassen Eid Abu-Sei'leek \\ Department of Physics, Faculty of Science, Zarqa University, Al-Zarqa, Jordan \\ Email:mabuseileek@zu.edu.jo,moh2hassen@yahoo.com
}

Received 3 January 2016; accepted 28 March 2016; published 31 March 2016

Copyright (C) 2016 by authors and Scientific Research Publishing Inc.

This work is licensed under the Creative Commons Attribution International License (CC BY). http://creativecommons.org/licenses/by/4.0/

(c) (i) Open Access

\begin{abstract}
Constrained spherical Hartree-Fock (CSHF) calculations under radial compression are presented for ${ }^{90} \mathrm{Zr}$ in a model space consisting of nine major oscillator shells. An effective baryon-baryon interaction which includes the $\Delta$ resonances is used. The nucleon-nucleon (N-N) interaction is Reid Soft Core (RSC) potential. The sensitivity of the results to the choice model space is examined. It is found that the nuclear system becomes more compressible when the model space is increased. The radial density and the number of $\Delta s$ are decreased by increasing model space. The results suggest that the behavior of single particle energies is independent of the model space.
\end{abstract}

\section{Keywords}

Nuclear Structure, Compressed Finite Nuclei, $\Delta$-Resonance

\section{Introduction}

The investigation of the properties for finite nucleus in its excitation state is very useful for understanding the products in heavy collision, barrier heights or saddle point configuration in nuclear fission. The Hartree-Fock (HF) theory [1]-[3] and more elaborate self-consistent theories [4] [5] have been widely and successfully applied to investigate various properties of nuclear structure.

One of the recent developments in the study of relativistic heavy-ion collisions is the identification of $\Delta$-rich nuclear systems. When the nuclear system is compressed by heavy-ion collision experiments, the $\Delta \mathrm{s}$ may constitute up to $10 \%$ of nuclear constituents [6]-[8].

In our earlier work, the excitation of $\Delta$-degree freedom in ${ }^{90} \mathrm{Zr}$ nucleus in (CSHF) was investigated [9], where the ground state properties of ${ }^{90} \mathrm{Zr}$ nucleus were examined in small model space that consisted of six major oscillator shells for $\mathrm{N}$ and six orbits for $\Delta$. The goal of this work is to reexamine these properties in large model space that consists of nine oscillator shells for $\mathrm{N}$ and ten orbits for $\Delta$. 
The $\Delta$ isobar is an important mode of nucleonic excitation [10] [11]. It is due to a resonance in pion-nucleon scattering, photopion and electropion production from nucleon. In nuclear structure, the $\Delta$ resonance is an agent for corrections in the traditional picture of the nucleus as a system of nucleons only [12] [13]. It is as a considered constituent of nucleus beside nucleons. The $\Delta$ isobar provides a mechanism for pion scattering, pion production, and pion absorption [14].

The theory of effective operators plays an important role in the modern approach to nuclear structure. The detailed calculations in Refs. [9] [15]-[18] demonstrated the effective Hamiltonian, $H_{\text {eff, }}$ and the calculation procedures in Refs. [19]-[22]. Based on the study presented in Ref. [9], the two-body matrix elements are scaled in the N-N sector to an optimal value of $\hbar \omega^{\prime}$, the oscillator energy for ${ }^{90} \mathrm{Zr}$ nucleus in the nine major oscillator shells with the ten delta orbits [19]-[22]. By using adjusting parameters: $\lambda_{1}, \lambda_{2}$, and $\hbar \omega^{\prime}$ it is possible to obtain such a fit to the equilibrium binding energy and $r_{r m s}$ radius. In this work, the adjusting parameters: $\lambda_{1}$, $\lambda_{2}$, and $\hbar \omega^{\prime}$ are: $0.999,1.047$, and 10.049 , respectively.

This paper is organized as follows: Section 2 specifies the results and discussions. Conclusions will be presented in Section 3.

\section{Results and Discussion}

In the previous studies [9] [23]-[25], the following quantities were calculated: the Hartree-Fock energy, the root mean square radius, the number of delta particles in the occupied orbits, the radial density distribution, the single-particle energies, and the occupation probability of the single-particle orbits in the case of small model space. In this work, more detailed results for ${ }^{90} \mathrm{Zr}$ nucleus are presented in order to examine its properties under static compression in larger model space consisting of nine major oscillator shells (excluding $\ell>5$ ) for nucleons and ten orbits for $\Delta$ s making a total of 47 baryons orbits.

\subsection{The Hartree-Fock Energies}

The bind energies $E_{H F}$ under compression using RSC potential are displayed in Figure 1 for ${ }^{90} \mathrm{Zr}$. The solid line represents results of the nucleon-only sector and the dashed line represents the results obtained when the $\Delta \mathrm{s}$ are added in the ten orbits: $0 p_{3 / 2}, \quad 0 p_{1 / 2}, \quad 0 d_{5 / 2}, \quad 0 d_{3 / 2}, \quad 0 f_{7 / 2}, 0 f_{5 / 2}, 1 p_{3 / 2}, 1 p_{1 / 2}, 0 g_{9 / 2}, 0 g_{7 / 2}$. Note that, in the CSHF approximation, the nucleons and delta may only mix in the $0 p_{3 / 2}, 0 p_{1 / 2}, 0 d_{5 / 2}, 0 d_{3 / 2}, 0 f_{7 / 2}$, $0 f_{5 / 2}, 1 p_{3 / 2}, 1 p_{1 / 2}, 0 g_{9 / 2}$, and $0 g_{7 / 2}$ states.

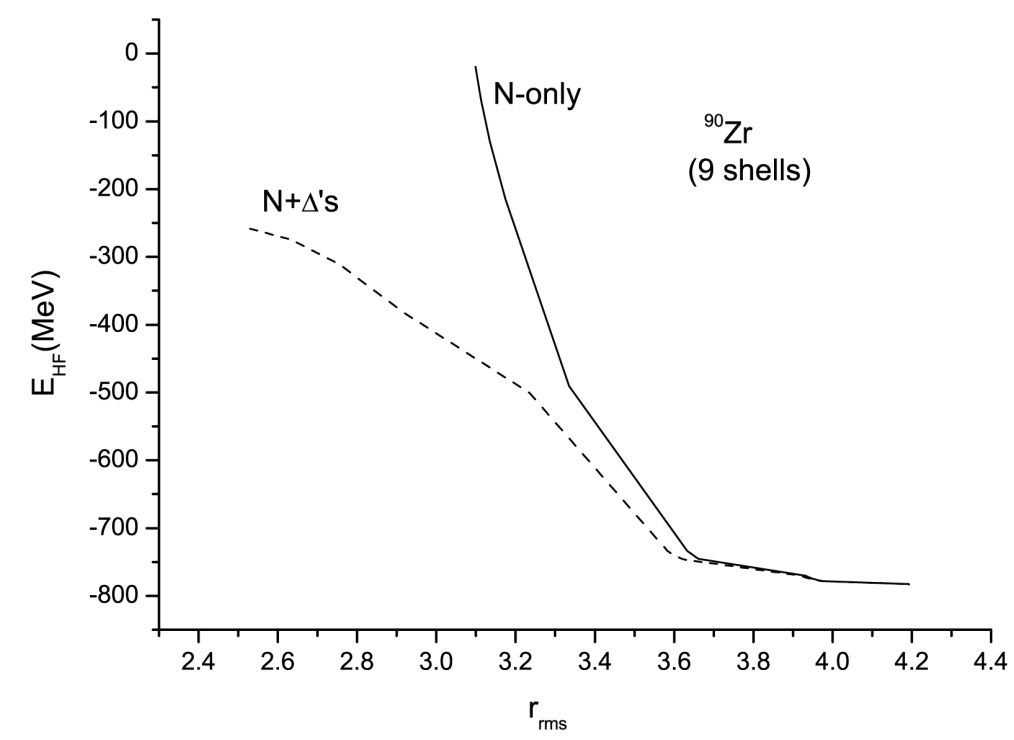

Figure 1. (CSHF ) energy as a function of the point mass $r_{r m s}$ for ${ }^{90} \mathrm{Zr}$ is evaluated in 9 major oscillator shells with $10 \Delta$-orbits. The dashed curve corresponds to CSHF, the full calculations including the $\Delta \mathrm{s}$, while the solid curve corresponding to CSHF with nucleons only. 
Figure 1 clearly shows that there is virtually no difference in the results with and without $\Delta \mathrm{s}$ at equilibrium. It is seen that without the $\Delta$-degree of freedom in the system, $E_{H F}$ increases steeply towards zero binding energy under compression. When the transition to $\Delta$ is allowed, the nucleus remains bound as density is increased to 4.54 of normal density. Considering only the nucleons, the volume (based on the root mean square radius) of nucleus decreases by about $40 \%$ and the binding energy will be about $250.36 \mathrm{MeV}$ with the inclusion of the $\Delta$-excitations. In other words, this shows about 587.49 MeV, and 337.13 MeV of excitation energy to achieve a $40 \%$ volume reduction in the nucleon-only results, and nucleons and $\Delta^{+}$'s results, respectively.

It appears from the above results that $337.13 \mathrm{MeV}$ of excitation energy is enough to reduce the volume by $40 \%$ and the energy by $43 \%$ more. The results show that there is a significant reduction in the static compression modulus for RSC static compressions which is reduced by including the $\Delta$ excitations. The consequence of this reduction is the softening of the nuclear equation of state at larger compression. To see the role of $\Delta$ in determining the equation of state, Figure 1 shows the dependence of the $E_{H F}$ on the compression characterized by the $r_{r m s}$. It can be seen that near equilibrium all curves agree. Also, the inclusion of $\Delta$ orbits tends to decrease of $E_{H F}$ for compressed nuclei. The role of the $\Delta \mathrm{s}$ is less significant as the $r_{\text {rms }}$ approaches the ground state value.

Figure 1 shows that as the static load force increases, the compression of nucleus with nucleons only is less than the other nucleus with nucleons and $\Delta \mathrm{s}$.

The difference between the results of the Hartree-Fock binding energy obtained in this work and those in Refs. [9] [23]-[25] is the size of the nucleon model space, the number of the $\Delta$ orbits included, different potentials and more compression. It is also worth mentioning that at equilibrium (no constraint) in ${ }^{90} \mathrm{Zr}$, it was not found any mixing between nucleon states and the $\Delta$ states. The same found in Refs. [9] [23]-[25], all curves of $E_{H F}$ are the same near equilibrium. This implies that results for the system at equilibrium don't depend on model space. In comparison with results in previous studies [9] [23]-[25], the current results are consistent with results obtained for ${ }^{90} \mathrm{Zr}$ for $E_{H F}$. The results of the Hartree-Fock energy without compression and the behavior of curves for nucleons only and nucleons $+\Delta s$ are the same for both the six and nine shells [9]. The static compression modulus is decreased significantly by increasing the nucleon model space. The current results display more compression than previous studies [9] [23]-[25]. It can be seen that the nuclear system becomes more compressible when the model space is increased. The results show that there is a significant reduction in the static compression modulus for RSC static compressions. It is reduced by including the $\Delta$ excitations. The consequence of this reduction is the softening of the nuclear equation of state at larger compression.

In terms of relativistic heavy-ion collisions, the nucleus that can penetrate more easily when the $\Delta$ degree of freedom becomes explicit is implied by Figure 1. Because of the limitations of the model space, the calculations for higher densities are more speculative. Nevertheless it can give us some idea about how the $\Delta$ population can be increased as the nucleus is compressed to higher densities accessible to relativistic heavy-ion collisions.

\subsection{The Number of $\Delta^{\prime} s$}

Figure 2 shows the role of the $\Delta^{\prime}$ 's as a function of compression. The total number of $\Delta^{\prime}$ s, $\Delta^{+}$'s and $\Delta^{0}$ 's are plotted separately.

In Figure 2, the number of deltas increases rapidly as volume decreases. When the nucleus volume is reduced to about $78 \%$ of its volume at equilibrium, the number of deltas is increased to about $5.56 \%$ of all constituents of

${ }^{90} \mathrm{Zr}$. It is interesting to note in Figure 2 that the number of $\Delta^{0} \mathrm{~s}$ and $\Delta^{+} \mathrm{s}$ are the same. In contrast to the previous findings [9] [24], the curves of number for $\Delta^{+} s$ and $\Delta^{0} s$ don't separate in the present work by increasing model space.

Although there is a rapid rise of the $\Delta$-population as compression increases, the change in the total number of $\Delta$ 's does not exceed 0.0556 in Figure 2. Is this a reasonable amount of $\Delta$ population? To examine this issue, it is interesting to note that there is a consistency of the amount of $\mathrm{N}-\Delta$ mixing with the amount of excitation energy exhibited with compression. That is, for example, when $0.0556 \Delta$ 's are presented, the excitation energy is in the order of $0.0556(\mathrm{M}-\mathrm{m}) \approx 16.52 \mathrm{MeV}$. Thus, on the scale of the unperturbed single-particle energies (including the rest mass of the nucleons and deltas), a substantial fraction of the compressive energy is delivered, through the $\mathrm{N}-\Delta$ and $\Delta-\Delta$ interactions to create more massive baryons in the lowest energy configuration of the nucleus. In other words, the number of $\Delta$ 's can be increased to about 5 at $r_{r m s}=2.53 \mathrm{fm}$ which corresponds to about 4.54 times the normal density. 


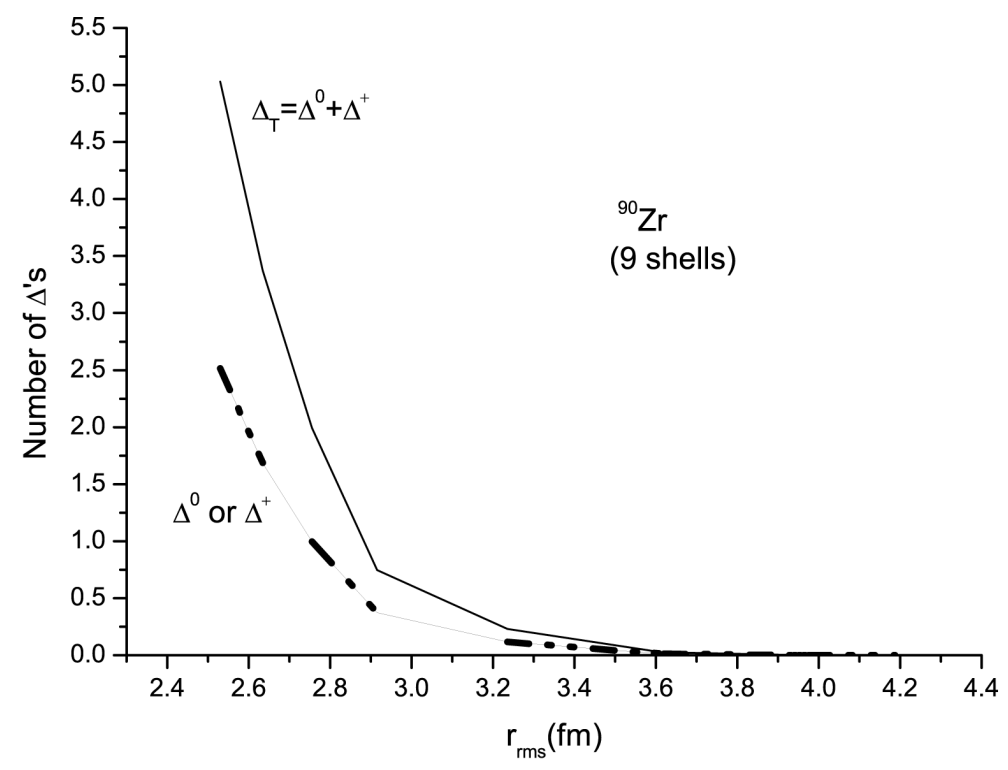

Figure 2. The number of $\Delta^{\prime}$ s as a function of $r_{r m s}$ for ${ }^{90} \mathrm{Zr}$ in 9 major shells model space. The upper curve is for the total number of $\Delta^{\prime}$ 's. The solid curve is for the number of $\Delta^{+}$, and the dashed curve is for $\Delta^{0}$.

Figure 2 shows that the number of created $\Delta$ s increases sharply when ${ }^{90} \mathrm{Zr}$ nucleus compressed to a volume of about 0.78 of its equilibrium size. However, at this nuclear density, which is 4.54 times the normal density, the percentage of nucleons converted to $\Delta$ is only about $5.56 \%$ in ${ }^{90} \mathrm{Zr}$. This result is consistent with our previous results [9] and the results of relativistic heavy-ion collisions [6]-[8] [26].

In comparison between our results with the previous results [23] [24], the current results show the number of $\Delta$ 's is decreased by increased model space.

One potential consequence of this result is that it could represent a collective mechanism for Subthreshold pion production. That is, in subthreshold pion production experiments between colliding nuclei where the bombarding energy per nucleon is below that needed to produce pions in N-N collisions. If the collisions produce isothermal compression, then the $\Delta$ 's are populated and relaxation could occur by the decay of the $\Delta$ to a nucleon and a pion. These calculations support a collective mechanism for subthreshold pion production.

\subsection{The Radial Density Distributions}

Figure 3 shows the radial density distributions for neutrons $\rho_{n}$, protons $\rho_{p}$, deltas $\rho_{\Delta}$, and their sum $\rho_{T}$ as a function of the radial distance from the center of the nucleus at large compression for ${ }^{90} \mathrm{Zr}$ nucleus in a model space of nine major oscillator shells with $\Delta$ excitation restricted to the ten orbits: $0 p_{3 / 2}, 0 p_{1 / 2}, 0 d_{5 / 2}, 0 d_{3 / 2}$, $0 f_{7 / 2}, 0 f_{5 / 2}, 1 p_{3 / 2}, 1 p_{1 / 2}, 0 g_{9 / 2}, 0 g_{7 / 2}$. The $\rho_{\Delta}$ is scaled by a factor of 10 to make it visible on the same scale as $\rho_{n}$ and $\rho_{p}$. In this figure, the neutron radial density is higher than the proton density at all values of $r$. This is due to Coulomb repulsion between the protons. The $\Delta$-radial density distribution, under high compression (point mass $r_{r m s}=3.54 \mathrm{fm}$ ), reaches a peak value of about 0.35 of the proton radial density at $r=2.80 \mathrm{fm}$. $\Delta$-mixing with the nucleons in the $0 p_{3 / 2}, \quad 0 p_{1 / 2}, \quad 0 d_{5 / 2}, \quad 0 d_{3 / 2}, \quad 0 f_{7 / 2}, 0 f_{5 / 2}, 1 p_{3 / 2}, 1 p_{1 / 2}, 0 g_{9 / 2}$, and $0 g_{7 / 2}$ orbits occurs and this explains the shape of the $\Delta$-radial distribution presented in Figure 3.

Figure 4 displays the radial density distributions of ${ }^{90} \mathrm{Zr}$ which is evaluated at about 0.16 reduced volume ( $r_{r m s}=2.29 \mathrm{fm}$ ). In this case, the $\Delta$-radial density distribution reaches a peak value of around 0.47 of the proton radial density at $2.40 \mathrm{fm}$.

In comparison between our results with the results of Ref. [9], the radial density distribution for $\Delta$ is decreased by increasing model space.

It can be seen from Figure 3, and Figure 4 that as compression increases, the total radial density increases and the radial density distribution of $\Delta \mathrm{s}$ increases sharply, whereas radial density of nucleons decreases sharply. This suggests that the less dense outer part of the nucleus initially responds to the external load more readily 


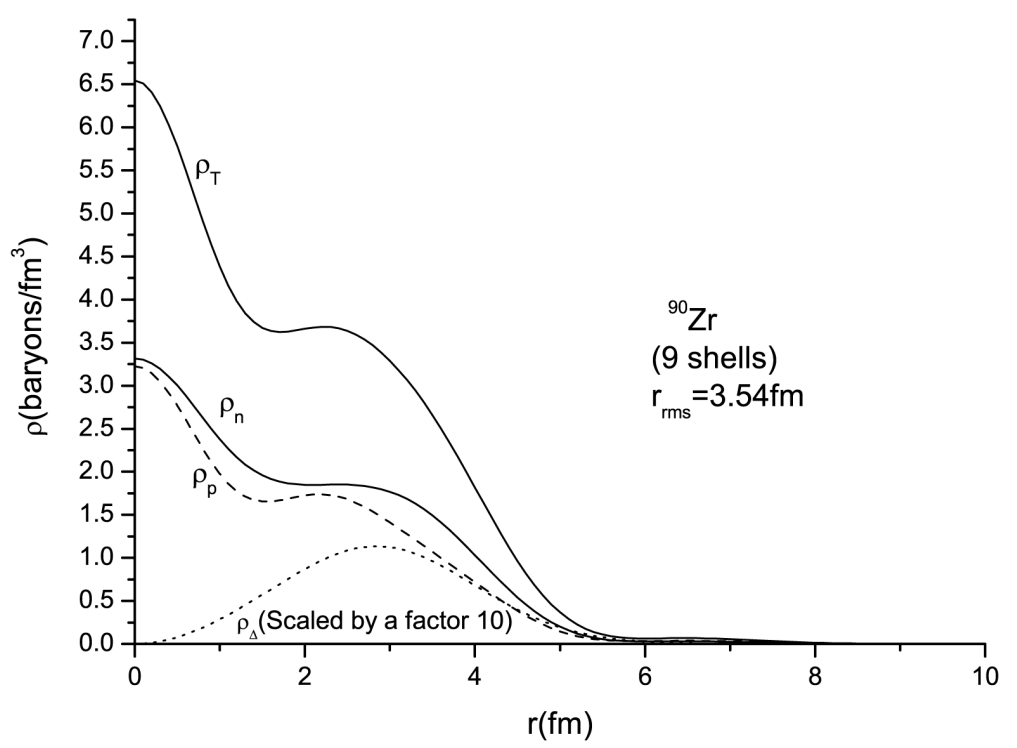

Figure 3. Total $\rho_{T}$, proton $\rho_{p}$ (dashed line), neutron $\rho_{n}$ (solid line) and delta $\rho_{\Delta}$ (dotted line) radial density distributions for ${ }^{90} \mathrm{Zr}$ at point mass radius $r_{r m s}=3.54 \mathrm{fm}$ in a model space of nine major oscillator shells.

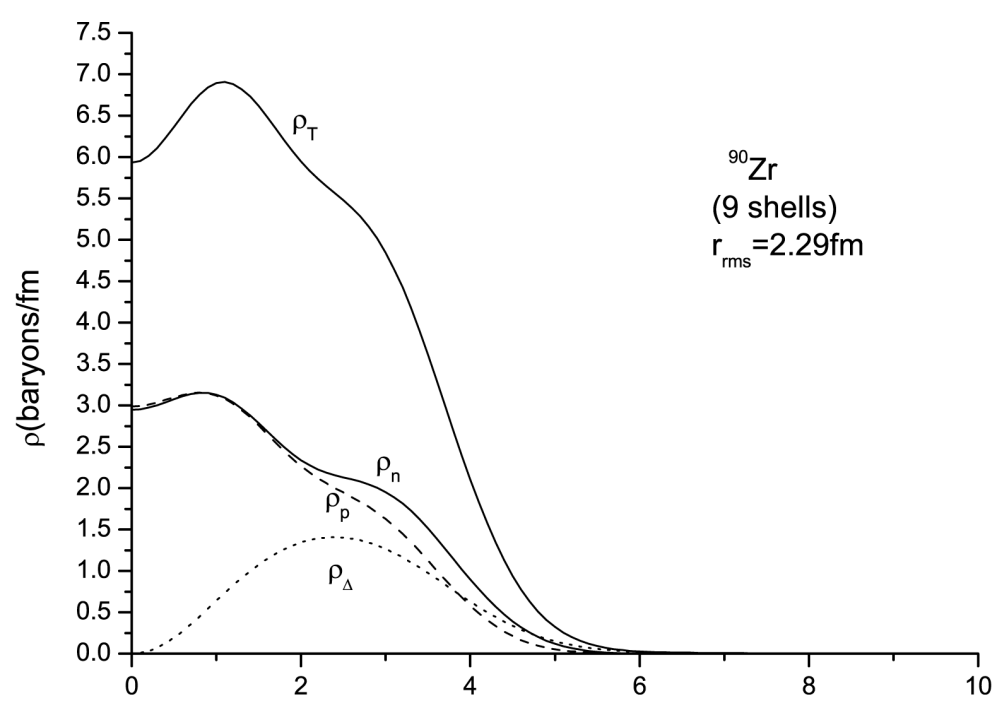

Figure 4. Total $\rho_{T}$, proton $\rho_{p}$ (dashed line), neutron $\rho_{n}$ (solid line), and delta $\rho_{\Delta}$ (dotted line) radial density distributions for ${ }^{90} \mathrm{Zr}$ at point mass $r_{r m s}=2.29 \mathrm{fm}$ in a model space of nine major oscillator shells.

than the inner part.

Clearly, the density in the interior rises relative to the interior density at equilibrium as the nucleus is compressed. This is in contrast to the behavior of the radial density on the outer-surface, where the radial density distribution is higher at equilibrium than the radial density when the static load is applied.

\subsection{Single Particle Energies}

Figure 5 displays the lowest self-consistent zero-change single particle energy levels as a function of $r_{\text {rms }}$. The orbits curved up as the load on the nucleus increases. This is because the kinetic energy of the baryons which is 


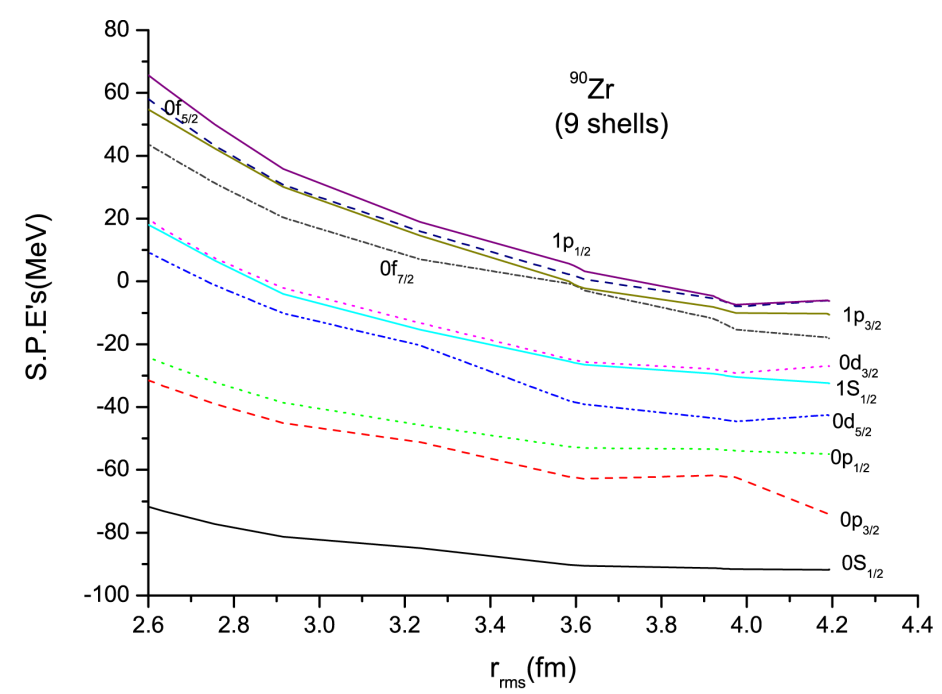

Figure 5. Single particle energy of lowest ten neutron states for ${ }^{90} \mathrm{Zr}$ in nine-oscillator shells as a function of $r_{r m s}$.

a positive quantity becomes more influential than the attractive mean field of the baryons.

The single particle energies increase linearly after $r_{r m s}=4.0 \mathrm{fm}$, they intersect at higher compression especially for higher orbits, and consequently the nucleus becomes unbound. The behavior of single particle energies is independent of the model space [9].

The single particle energy spectrum also sees the gaps between the shells. As the nucleus is compressed, the single particle level ordering and gaps are preserved. The general trend exhibited the single particle energies (except the deepest bound orbital which actually drops with compression) shift to higher energies as the nucleus is compressed. The curvature goes up more and more as the orbital becomes closer to the surface. This implies that the surface is more responsive to compression than the interior of nucleus.

Moreover, single particle spectrum is generated entirely from the underlying microscopic Hamiltonian. This is a remarkable result since the calculated spectrum follows the expected ordering of the phenomenological shell model in the dominantly nucleons orbits. The spectrum also exhibits clearly visible gaps between the shells. As the nucleus is compressed, the single particle level ordering and the gaps are preserved. It is worth noting that the orbits closest to zero single-particle energy are more sensitive to compression. This means that these orbits are more responsive to compression than the interior orbits of nucleus. The general trend of single-particle energies exhibits a shift to higher energies as the nucleus is compressed.

The behavior of single particle energy levels is in good agreement with the orbital ordering of the standard shell model. The gap is very clear between the shells. The splitting of the levels in each shell is an indication that orbital-spin (L-S) coupling is strong enough in RSC potential, i.e., L-S coupling becomes stronger as the static load on the nucleus increases. When the nucleus is compressed, the splitting of the orbits becomes more noticeable especially in delta orbits.

\section{Conclusions}

The ground state properties of the neutron-rich spherical ${ }^{90} \mathrm{Zr}$ nucleus have been investigated by using a realistic effective baryon-baryon Hamiltonian in the CSHF approximation. In model space consisting of nine major oscillator shells with $\Delta$ resonances of nucleons restricted to $\Delta$ orbits, the sensitivity of the results to the choice model space is examined.

It is found that the nuclear system becomes more compressible when the model space is increased. The radial density and the number of $\Delta \mathrm{s}$ are decreased by increasing model space. The behavior of single particle energies is found to be independent of the model space.

Future outlooks of this research are: we will increase the model space; a modified version of RSC should be used; thermal properties of finite nuclei can be incorporated in this model; a more general treatment should relax 
this restriction in order to allow for other mixing, e.g., (n, $\left.\Delta^{-}\right),\left(\mathrm{p}, \Delta^{++}\right),\left(\Delta^{0}, \Delta^{-}\right),\left(\Delta^{+}, \Delta^{++}\right)$. Deformed nuclei can be studied by this model; relativistic effects can be also investigated and semi-classical descriptions of heavy-ion reaction at intermediate energies can be investigated.

\section{Acknowledgements}

The author acknowledges support for this research from the Deanship of Research at Zarqa University/Jordan.

\section{References}

[1] Ripka, G., Baranger, M. and Vogt, E. (1968) Advances in Nuclear Physics. Vol. 1, Phenum.

[2] Guleria, N., Dhiman, S.K. and Shyam, R. (2012) A Study of $\Lambda$ Hypernuclei within the Skyrme-Hartree-Fock Model. Nuclear Physics A, 886, 71-91. http://dx.doi.org/10.1016/j.nuclphysa.2012.05.005

[3] Sekizawa, K. and Yabana, K. (2013) Time-Dependent Hartree-Fock Calculations for Multinucleon Transfer Processes in ${ }^{40,48} \mathrm{Ca}+{ }^{124} \mathrm{Sn},{ }^{40} \mathrm{Ca}+{ }^{208} \mathrm{~Pb}$, and ${ }^{58} \mathrm{Ni}+{ }^{208} \mathrm{~Pb}$ Reactions. Ar-xiv: 1303.0552 [nucl-th].

[4] Ripka, G. and Porneuf, M. (1976) Proceedings of the International Conference on Nuclear Self-Consistent Fields, Organised and Held at the International Centre for Theoretical Physics Trieste, 24-28 February 1975. http://www.iberlibro.com/servlet/BookDetailsPL?bi=8320769375\&searchurl=tn\%3Dtheoretical\%2520nuclear\%2520p hysics

[5] Gnezdilov, N.V., Borzov, I.N., Saperstein, E.E. and Tolokonnikov, S.V. (2014) Arxiv:1401.1319v4 [nucl-th].

[6] Mosel, U. and Metag, V. (1993) Evidence for Resonance Matter at SIS. Nuclear Physics News, 3, 25-27. http://dx.doi.org/10.1080/10506899308221163

[7] Xiong, L., Wu, Z.G., Ko, C.M. and Wu, J.Q. (1990) Dielectron Production from Nucleus-Nucleus Collisions. Physical Review A, 512, 772.

[8] Hofmann, M., Mattiello, R., Sorge, H., Stöcker, H. and Greiner, W. (1995) Stopped $\Delta$-Matter Source in Heavy-Ion Collisions at $10 \mathrm{GeV} /$ Nucleon? Physical Review C, 51, 2095.

[9] Abu-Seileek, M.H. (2011) Resonances-Excitation Calculation Studies Investigation of $\Delta(3,3)$ in Ground State of ${ }^{90} \mathrm{Zr}$ Cold Finite Heavy Nucleus at Equilibrium and under Large Compression. Communications in Theoretical Physics, 55, 115. http://dx.doi.org/10.1088/0253-6102/55/1/22

[10] Ericson, T. and Weise, W. (1988) Pions and Nuclei. Clarendon Press.

[11] Ring, P. and Schuch, P. (1980) The Nuclear Many Body Problem. Springer, Berlin. http://dx.doi.org/10.1007/978-3-642-61852-9

[12] Green, A.M. (1976) Nucleon Resonance in Nuclei. Reports on Progress in Physics, 39, 1109. http://dx.doi.org/10.1088/0034-4885/39/12/001

[13] Sauer, P.U. (1986) Microscopic Nuclear Structure with Sub-Nucleonic Degrees of Freedom. Progress in Particle and Nuclear Physics, 16, 35-102. http://dx.doi.org/10.1016/0146-6410(86)90002-5

[14] Valcare, A., Fernnder, F., Garcilazo, H., Pea, M.T. and Sauer, P.U. (1996) Effects of a Quark-Model-Based Nucleon- $\Delta$ Potential on the Two-Nucleon System above Pion Threshold. Physical Review C, 49, 1799. http://dx.doi.org/10.1103/PhysRevC.49.1799

[15] Abu-Seileek, M.H. and Hasan, M.A. (2010) $\Delta$-Resonances in Ground State Properties of $20^{40}$ Ca Spherical Cold Finite Nucleus at Equilibrium and under Compression. Communications in Theoretical Physics, 54, 339. http://dx.doi.org/10.1088/0253-6102/54/2/25

[16] Abu-Seileek, M.H. (2011) Hartree-Fock Calculation Studies Investigation of $\Delta(3,3)$ Resonances in the Ground State of Compressed Heavy Spherical Finite Nucleus ${ }^{132}$ Sn. International Journal of Pure and Applied Physics, 7, 73-87.

[17] Abu-Seileek, M.H. (2011) Investigation of $\Delta(3,3)$ Resonance Effects on the Properties of Neutron-Rich Double Magic Spherical Finite Nucleus, ${ }^{132} \mathrm{Sn}$, in the Ground State and under Compression. Pramana, 76, 573-589. http://dx.doi.org/10.1007/s12043-011-0063-x

[18] Abu-Seileek, M.H. (2011) Delta Excitation Calculation Studies in the Ground State of the Compressed Finite Heavy Doubly-Magic Nucleus ${ }^{100}$ Sn. Turkish Journal of Physics, 35, 273.

[19] Abu-Seileek, M.H. (2010) Delta Excitation in Compressed Neutron-Rich Double Magic Spherical Finite Nucleus ${ }^{132}$ Sn. Nuclear Physics Review, 27, 399-410.

[20] Abu-Seileek, M.H. (2011) Delta Excitation Calculation Studies in Compressed Finite Spherical Nucleus ${ }^{40}$ Ca. Nuclear Physics Review, 28, 416-422.

[21] Abu-Seileek, M.H. (2011) Doubly-Magic ${ }^{100}$ Sn Nucleus with Delta Excitation under Compression. Journal of the 
Physical Society of Japan, 80, Article ID: 104201. http://dx.doi.org/10.1143/JPSJ.80.104201

[22] Abu-Sei’leek, M.H. (2014) Neutron-Rich ${ }^{208} \mathrm{~Pb}$ Nucleus with Delta Excitation under Compression. Turkish Journal of Physics, 38, 253-260. http://dx.doi.org/10.3906/fiz-1402-4

[23] Hasan, M.A., Lee, T.S.H. and Vary, J.P. (1997) Mean-Field Calculation of Compressed Finite Nuclei with $\Delta$ Excitations. Physical Review C, 56, 3063-3069. http://dx.doi.org/10.1103/PhysRevC.56.3063

[24] Hasan, M.A., Vary, J.P. and Lee, T.S.H. (2001) Medium-Mass Nuclei with $\Delta$ Excitations under Compression. Physical Review C, 64, Article ID: 024306. http://dx.doi.org/10.1103/PhysRevC.64.024306

[25] Hasan, M.A. and Vary, J.P. (1994) Delta Excitations in Compressed Finite Nuclei. Physical Review C, 50, $202-212$. http://dx.doi.org/10.1103/PhysRevC.50.202

[26] Xing, Y.-Z., Zheng, Y.-M., Pornrad, S., Yan, Y.-P. and Chinorat, K. (2009) Differential Directed Flow of $K^{+}$Meson within Covariant Kaon Dynamics. Chinese Physics Letters, 26, Article ID: 022501. http://dx.doi.org/10.1088/0256-307X/26/2/022501 